\title{
Levonorgestrel-releasing intrauterine system versus endometrial ablation for heavy menstrual bleeding
}

\author{
Pleun Beelen, MD¹; Marian J. van den Brink, MD¹; Malou C. Herman, PhD; Peggy M. A. J. Geomini, PhD; Janny H. Dekker, PhD; \\ Ruben G. Duijnhoven, PhD; Nienke Mak, MD; Hannah S. van Meurs, PhD; Sjors F. Coppus, PhD; \\ Jan Willem van der Steeg, PhD; Heleen P. Eising, MD; Diana S. Massop-Helmink, MD; Ellen R. Klinkert, PhD; \\ Theodoor E. Nieboer, PhD; Anne Timmermans, PhD; Lucet F. van der Voet, PhD; Sebastiaan Veersema, Prof PhD; \\ Nicol A. C. Smeets, PhD; Joke M. Schutte, PhD; Marchien van Baal, PhD; Patrick M. Bossuyt, Prof PhD; \\ Ben Willem J. Mol, Prof PhD; Marjolein Y. Berger, Prof PhD; Marlies Y. Bongers, Prof PhD
}

BACKGROUND: Heavy menstrual bleeding affects the physical functioning and social well-being of many women. The levonorgestrelreleasing intrauterine system and endometrial ablation are 2 frequently applied treatments in women with heavy menstrual bleeding.

OBJECTIVE: This study aimed to compare the effectiveness of the levonorgestrel-releasing intrauterine system with endometrial ablation in women with heavy menstrual bleeding.

STUDY DESIGN: This multicenter, randomized controlled, noninferiority trial was performed in 26 hospitals and in a network of general practices in the Netherlands. Women with heavy menstrual bleeding, aged 34 years and older, without a pregnancy wish or intracavitary pathology were randomly allocated to treatment with either the levonorgestrelreleasing intrauterine system (Mirena) or endometrial ablation, performed with a bipolar radiofrequency device (NovaSure). The primary outcome was blood loss at 24 months, measured with a Pictorial Blood Loss Assessment Chart score. Secondary outcomes included reintervention rates, patient satisfaction, quality of life, and sexual function.

RESULTS: We registered 645 women as eligible, of whom 270 women provided informed consent. Of these, 132 women were allocated to the levonorgestrel-releasing intrauterine system (baseline Pictorial Blood Loss Assessment Chart score, 616) and 138 women to endometrial ablation (baseline Pictorial Blood Loss Assessment Chart score, 630). At 24 months, mean Pictorial Blood Loss Assessment Chart scores were 64.8 in the levonorgestrel-releasing intrauterine system group and 14.2 in the endometrial ablation group (difference, 50.5 points; 95\% confidence interval, 4.3-96.7; noninferiority, $P=.87$ [25 Pictorial Blood Loss Assessment Chart point margin]). Compared with 14 women (10\%) in the endometrial ablation group, 34 women (27\%) underwent a surgical reintervention in the levonorgestrel-releasing intrauterine system group (relative risk, 2.64; 95\% confidence interval, 1.49-4.68). There was no significant difference in patient satisfaction and quality of life between the groups.

CONCLUSION: Both the levonorgestrel-releasing intrauterine system and endometrial ablation strategies lead to a large decrease in menstrual blood loss in women with heavy menstrual bleeding, with comparable quality of life scores after treatment. Nevertheless, there was a significant difference in menstrual blood loss in favor of endometrial ablation, and we could not demonstrate noninferiority of starting with the levonorgestrelreleasing intrauterine system. Women who start with the levonorgestrelreleasing intrauterine system, a reversible and less invasive treatment, are at an increased risk of needing additional treatment compared with women who start with endometrial ablation. The results of this study will enable physicians to provide women with heavy menstrual bleeding with the evidence to make a well-informed decision between the 2 treatments.

Key words: excessive uterine bleeding, intrauterine device, menorrhagia, Mirena, NovaSure

\section{Introduction}

Around $30 \%$ of women worldwide experience heavy menstrual bleeding (HMB). It poses a major burden because it negatively influences physical activity, work productivity, and sexual life and is

Cite this article as: Beelen $\mathrm{P}$, van den Brink MJ, Herman $M C$, et al. Levonorgestrel-releasing intrauterine system versus endometrial ablation for heavy menstrual bleeding. Am J Obstet Gynecol 2021;224:187.e1-10.

\section{2-9378}

(C) 2020 The Authors. Published by Elsevier Inc. This is an open access article under the CC BY license (http:// creativecommons.org/licenses/by/4.0/). https://doi.org/10.1016/j.ajog.2020.08.016

Click Supplemental Materials under article title in Contents at ajog a common reason for consulting a gynecologist. ${ }^{1,2}$ In view of its high prevalence and major impact on the quality of life (QoL), effective treatment is of utmost importance.

The National Institute for Health and Care Excellence guideline on HMB recommends the levonorgestrel-releasing intrauterine system (LNG-IUS; Mirena, Bayer HealthCare Pharmaceuticals, Berlin, Germany) $52 \mathrm{mg}$ as the treatment of first choice. ${ }^{2}$ Randomized controlled trials (RCTs) comparing the LNG-IUS with oral medication have shown significantly reduced menstrual bleeding and increased QoL in favor of the LNGIUS. ${ }^{3,4}$ Nevertheless, approximately $40 \%$ of women discontinue the LNG-IUS within 2 years because of a lack of effectiveness, irregular or prolonged bleeding, abdominal pain, or progestogenic side effects. ${ }^{3,5}$

Alternative surgical options for treating $\mathrm{HMB}$ in women without future pregnancy wish are endometrial ablation (EA) and hysterectomy. Hysterectomy is a definitive solution, but it is an invasive option, with a risk of serious complications. ${ }^{6}$ The EA procedure is a minimally invasive alternative to hysterectomy that aims to destroy or remove the endometrial tissue. Bipolar radiofrequency EA results in amenorrhea in $50 \%$ of women, satisfaction rates between $80 \%$ and $96 \%$, and reported reintervention rates around $10 \% .^{7-10}$ 


\section{AJOG at a Glance}

Why was this study conducted?

The levonorgestrel-releasing intrauterine system (LNG-IUS) and endometrial ablation (EA) are 2 frequently used treatments in women with heavy menstrual bleeding (HMB). Counseling women with $\mathrm{HMB}$ is challenging because it is unclear which of these 2 treatments is more effective.

\section{Key findings}

Both treatment strategies lead to a large decrease in menstrual blood loss, but the EA strategy results in the least amount of menstrual blood loss. Patient satisfaction, quality of life, and sexual functioning are comparable between both strategies. Women treated with the LNG-IUS are at an increased risk of receiving another intervention to achieve the desired result.

\section{What does this add to what is known?}

These results on multiple outcomes enable physicians to provide women with complete information so that they can make a well-informed decision about treatment.

The LNG-IUS seems superior to oral medication, but the evidence on the effectiveness of the LNG-IUS compared with EA is inconclusive. Previous studies show conflicting results on bleeding scores, satisfaction, and reinterventions. ${ }^{6,11-19}$ Moreover, these studies suffered from a risk of attrition bias and had relatively small sample sizes, and most studies had short followup periods. These deficiencies hamper firm conclusions about the choice for either the LNG-IUS or EA, which makes counseling complicated.

Because of the advantages of the LNGIUS over EA in terms of invasiveness, reversibility, contraceptive effect, and feasibility in general practice, we performed a multicenter RCT to investigate if a strategy starting with the LNG-IUS is noninferior to a strategy starting with bipolar radiofrequency EA.

\section{Materials and Methods}

\section{Study design}

This multicenter RCT was performed between 2012 and 2016 in 26 hospitals collaborating in the Dutch Consortium for Studies in Women's Health and Reproduction and in a network of general practices in the Netherlands. The study was conducted according to the principles of the Declaration of Helsinki and approved by the ethics committee of the Academic Medical Center in
Amsterdam, Netherlands (registration number 2011_372). The study was registered prospectively in the Netherlands Trial Register (NL2842). Full details of the protocol have been published previously. ${ }^{20}$

After consultation with the Data and Safety Monitoring Board (DSMB) on September 19, 2012, an adjustment to the protocol was made. The board advised to change the primary outcome from satisfaction to menstrual blood loss (measured with the Pictorial Blood Loss Assessment Chart [PBAC] score). The reason for this change was that satisfaction is a subjective outcome that depends on women's expectations about the treatment and is therefore not an objective outcome to measure the difference in treatment effect. The amount of menstrual blood loss was considered as a more objective outcome to measure the difference in effectiveness. The change in primary outcome was approved by the ethics committee in March 2013. At that time, 24 women had been included in the study whose collected data were not accessible by the researchers.

Women were eligible if they had HMB, with a PBAC score exceeding 150 points. Women could have received previous treatment (oral) for HMB. Women were excluded if they were younger than 34 years; had a pregnancy wish; had an abnormal cervix cytology in the past 5 years; had intracavitary fibroids, polyps, or large intramural fibroids; had a uterus length of $>10 \mathrm{~cm}$; or had a uterine size $>10$ weeks of gestation measured by either a transvaginal ultrasound or bimanual vaginal examination, depending on the setting (general practice or gynecology department).

Eligible women were identified by the gynecologists in the participating hospitals or by the participating general practitioners. Research nurses or treating physicians obtained written informed consent from all participating women before randomization.

\section{Randomization and blinding}

Consenting women were randomly allocated in a 1:1 ratio to 1 of 2 treatment arms by research nurses at the local centers, using an internet-based randomization module. Randomization was performed in blocks, with a variable block size of 2 or 4 , and was stratified for recruitment by the general practitioner or gynecologist. Because of the nature of the intervention, it was not possible to blind the participants and their treating physicians for the allocated treatment.

\section{Interventions}

The LNG-IUS (Mirena, Bayer HealthCare Pharmaceuticals) is a T-shaped device placed into the endometrial cavity, containing a total of $52 \mathrm{mg}$ of levonorgestrel, releasing $20 \mu \mathrm{g} / \mathrm{d}$. The system is licensed up to 5 years of use. ${ }^{21}$ Insertion of the LNG-IUS is an outpatient procedure, which can be performed without anesthesia. In women allocated to the LNG-IUS group, the device was inserted by a gynecologist, resident, or general practitioner skilled in inserting the LNG-IUS.

The EA procedure was performed with a bipolar radiofrequency device: NovaSure system (NovaSure, Hologic, Marlborough, MA), a nonresectoscopic ablation technique that destroys the uterine endometrium using radiofrequency electrical energy. ${ }^{22,23}$ In women allocated to EA group, the procedure was performed by a gynecologist or resident experienced in treatment with the NovaSure device. The 


\section{FIGURE 1}

\section{Trial profile}

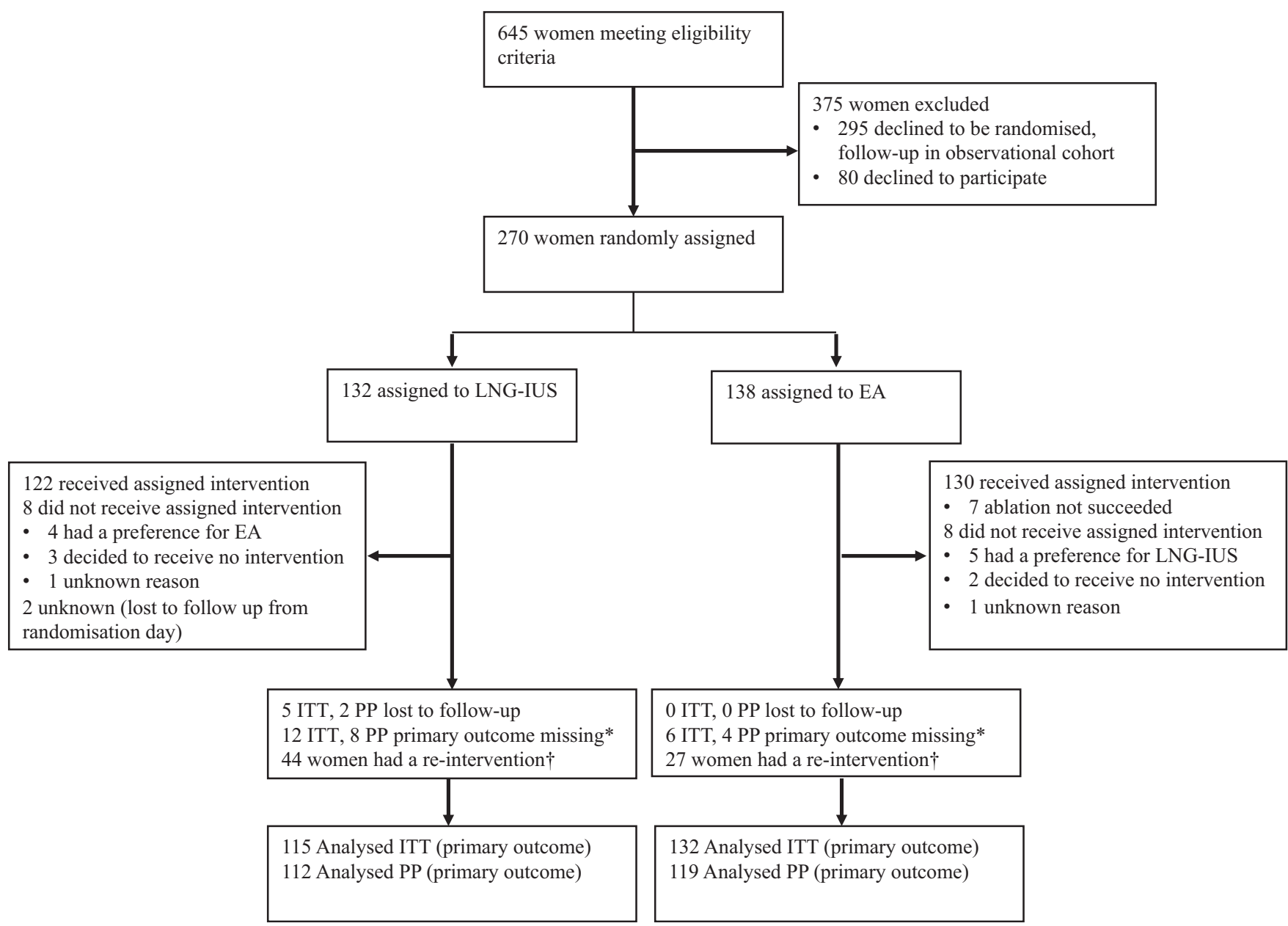

*Women excluded for primary analysis but included for secondary analyses. See Table 3 for type of, number of, and reason for reinterventions.

$E A$, endometrial ablation; ITT, intention to treat; $L N G-I U S$, levonorgestrel-releasing intrauterine system; $P P$, per protocol.

Beelen et al. Levonorgestrel-releasing intrauterine system versus endometrial ablation for heavy menstrual bleeding. Am J Obstet Gynecol 2021.

procedure was performed in the office under local anesthetics or conscious sedation or in the operating room under general or spinal anesthesia depending on the local setting.

\section{Outcomes}

The primary outcome was blood loss at 24 months after randomization, measured with the PBAC score according to Higham. ${ }^{24}$ To calculate this score, women were asked to record their menstrual blood loss for 1 month, counting the number of super tampons or pads used each day. The total score was calculated using a score of 1 for each lightly saturated tampon, 5 for moderately saturated tampons, and 10 for completely saturated tampons. For pads, the scores were 1,5 , and 20 , respectively. ${ }^{24}$ Secondary outcome measures were controlled bleeding, defined as a PBAC score not exceeding 75 points, ${ }^{6}$ complications and reinterventions within 24 months after randomization, amenorrhea, spotting, dysmenorrhea, presence of clots, duration of blood loss, satisfaction with treatment (measured with a 5point Likert scale), QoL (measured with the general 36-Item Short Form Health Survey [SF-36] questionnaire and the disease-specific Menorrhagia MultiAttribute Scale [MMAS, also known as Shaw Menorrhagia Questionnaire]), ${ }^{25-28}$ and sexual function (measured with the Female Sexual Function Index [FSFI] and Female Sexual Distress Scale [FSDS]). ${ }^{29,30}$

Women were required to complete the questionnaires before treatment and at $3,6,12$, and 24 months after randomization. Research nurses at the local centers were responsible for collecting baseline and follow-up data. Serious adverse events (SAEs) were monitored by the local investigator and were reported within 7 days after notification. In addition, data on adverse events were collected from medical records by the participating research nurses and from the participant questionnaires. 


\section{TABLE 1 \\ Baseline characteristics}

\section{Characteristic}

Age, mean (SD)

LNG-IUS ( $\mathrm{n}=132)$

$E A(n=138)$

$\mathrm{BMI}^{\mathrm{a}}{ }^{\mathrm{a}}$ mean (SD)

44.7 (4.6)

$45.3(4.9)$

Vaginal deliveries

\begin{tabular}{lll}
\hline 0 & $25(20.0)$ & $23(17.0)$ \\
\hline 1 & $15(12.0)$ & $26(19.0)$ \\
\hline$>1$ & $84(68.0)$ & $87(64.0)$ \\
\hline Cesarean delivery & $30(23.0)$ & $23(17.0)$
\end{tabular}

Previous uterus surgery

\begin{tabular}{lcc}
\hline Myomectomy & $7(5.3)$ & $11(8.0)$ \\
\hline Polypectomy & $4(3.0)$ & $5(3.6)$ \\
\hline Duration of HMB (mo), ${ }^{\text {a }}$ median (IQR) & $12.0(6.0-28.0)$ & $12.0(5.0-24.0)$
\end{tabular}

Duration of HMB (mo), ${ }^{\text {a }}$ median (IQR)

Previous treatment ${ }^{b}$

Tranexamic acid

$19(14.0)$

$20(14.0)$

NSAIDs

7 (5.3)

$10(7.2)$

Oral contraceptives

$33(25.0)$

$32(23.0)$

Progestogens

17 (13.0)

$14(10.0)$

LNG-IUS

$3(2.3)$

5 (3.6)

GnRH agonist

$1(0.8)$

$0(0.0)$

NuvaRing

$0(0.0)$

$1(0.7)$

\section{Anticoagulants}

Platelet aggregation inhibitors

Vitamin $\mathrm{K}$ antagonists

2 (1.5)

$1(0.7)$

$3(2.3)$

$1(0.7)$

\section{Current hormonal medication}

Oral contraceptives

Progestogens

$19(14.0)$

$17(12.0)$

$4(3.0)$

$5(3.6)$

Dysmenorrhea $^{\mathrm{a}}$

\section{Presence of dysmenorrhea}

Mild

\section{Moderate}

Severe

Duration of menstruation (d), median (IQR)

PBAC score, mean (SD)

Values are given as number of women (percentage), unless otherwise indicated.

$B M I$, body mass index; $E A$, endometrial ablation; $G n R H$, gonadotropin-releasing hormone; $H M B$, heavy menstrual bleeding; $I Q R$, interquartile range; $L N G-I U S$, levonorgestrel-releasing intrauterine system; NSAID, nonsteroidal antiinflammatory drug; NuvaRing, etonogestrel and ethinylestradiol vaginal ring; PBAC, Pictorial Blood Loss Assessment Chart; SD, standard deviation.

a Values were missing in some women; ${ }^{b}$ Women could have received $\geq 1$ previous treatment.

Beelen et al. Levonorgestrel-releasing intrauterine system versus endometrial ablation for heavy menstrual bleeding. Am J Obstet Gynecol 2021.

\section{Statistical methods}

We expressed the effectiveness of the LNG-IUS relative to EA in terms of the difference in mean PBAC score at 24 months, using $95 \%$ confidence intervals (CIs) to express statistical uncertainty. Our primary analysis was an intentionto-treat (ITT) analysis with the hypothesis that the LNG-IUS would be noninferior to EA. Previous studies in women with $\mathrm{HMB}$ have shown that a 50point difference in PBAC score between treatments is clinically meaningful. ${ }^{14,15,24,31}$ In an expert panel of 12 members, consisting of gynecologists, general practitioners, and methodologists, a difference of maximally 25 points was selected for expressing noninferiority. This margin was based on results from previous studies and clinical judgment, in which the practical consequence of the difference in PBAC score, translating it to the number of tampons or pads being used, was taken into account. Noninferiority of the LNG-IUS treatment would be shown if the upper limit of the $95 \%$ CI for the difference in PBAC score did not exceed the predetermined noninferiority margin of 25 points, with a corresponding $P$ value of $<.05$. Accordingly, the statistical null hypothesis for the trial was inferiority of the LNG-IUS treatment compared with EA treatment. With a $P$ value of $<.05$, this null hypothesis would be rejected, and the alternative-noninferiority of LNG-IUS treatment-accepted.

On the basis of previous studies, we estimated the mean PBAC score at 24 months after randomization to be 50 points in the LNG-IUS group and 40 points in the EA group, with a standard deviation of 40 points. ${ }^{4,14-16,31,32}$ Allowing for a dropout rate of up to $15 \%$ and using an alpha of $5 \%$ and a power of $80 \%$, we aimed to recruit 266 women in total (133 women per arm).

After first analyzing the data, the distribution of the PBAC scores at 24 months follow-up was found to be highly skewed with many zeros. This rendered the assumptions of the initially planned analysis, relying on the mean difference and $t$ test statistic, to be no longer valid. Therefore, we calculated the $95 \%$ CIs using bootstrapping $(10.000$ replications) and also relied on bootstrapping to test the noninferiority hypothesis. Because of the skewed distribution, we additionally used a zeroinflated negative-binomial model to evaluate a difference in the primary outcome measure. 
TABLE 2

Menstruation pattern at 24 months of follow-up

\begin{tabular}{|c|c|c|c|c|c|}
\hline Variable & LNG-IUS $(n=115)$ & $E A(n=132)$ & Effect (RR) & $95 \% \mathrm{Cl}$ & $P$ value \\
\hline PBAC score, mean (SD) & $64.8(251.0)$ & $14.2(43.4)$ & $50.50^{\mathrm{a}}$ & $4.3-96.7^{b}$ & $.87^{\circ}$ \\
\hline Amenorrhea & $67(58.0)$ & $89(67.0)$ & 0.86 & $0.71-1.05$ & \\
\hline$\leq 75$ & $100(87.0)$ & $124(94.0)$ & 0.93 & $0.85-1.01$ & \\
\hline$>75-150$ & $9(7.8)$ & $6(4.5)$ & 1.72 & $0.63-4.69$ & \\
\hline$>150$ & $6(5.2)$ & $2(1.5)$ & 3.44 & $0.71-16.7$ & \\
\hline Duration of blood loss $\leq 7 \mathrm{~d}$ & $105(91.0)$ & $126(95.0)$ & 0.96 & $0.89-1.02$ & \\
\hline Spotting & $21(18.0)$ & $18(14)$ & 1.34 & $0.75-2.39$ & \\
\hline Presence of clots & $7(6.1)$ & $4(3.0)$ & 2.01 & $0.60-6.69$ & \\
\hline Presence of dysmenorrhea & $27(23.0)$ & $19(14.0)$ & 1.63 & $0.96-2.77$ & \\
\hline Mild & $11(9.6)$ & $6(4.5)$ & - & - & \\
\hline Moderate & $10(8.7)$ & $7(5.3)$ & - & - & \\
\hline Severe & $6(5.2)$ & $6(4.5)$ & - & - & \\
\hline \multicolumn{6}{|c|}{ Intention-to-treat analysis. Values are given as number of women (percentage), unless otherwise indicated. } \\
\hline \multicolumn{6}{|c|}{$C l$ confidence interval; $E A$, endometrial ablation; $L N G-U S$, levonorgestrel-releasing intrauterine system; $P B A C$, Pictorial Blood Loss Assessment Chart; $R R$, relative risk; $S D$, standard deviation. } \\
\hline \multicolumn{6}{|c|}{${ }^{\mathrm{a}}$ Mean difference; ${ }^{\mathrm{b}} \mathrm{Cl}$ calculated using bootstrapping (2.5th percentile and 97.5 th percentile); ${ }^{\mathrm{c}} P$ value for noninferiority. } \\
\hline
\end{tabular}

Because the analysis of a noninferiority trial in the ITT study group may bias the results toward equivalence, we performed an additional perprotocol analysis of the primary outcome measure, including all women who received the allocated treatment.

Relative risks (RRs) or mean differences were estimated for secondary outcomes at different time points, with 95\% CIs. Secondary outcomes were analyzed according to the ITT principle. We plotted Kaplan-Meier curves for the time between randomization and first surgical reintervention and evaluated differences using log-rank statistics. Data on QoL and sexual function scores were analyzed using a generalized linear model for repeated measures (generalized estimating equation).

For the primary outcome, no imputation was used for missing data. Missing data on secondary outcomes were not imputed if a complete questionnaire at a follow-up point was missing.

All statistical tests were performed with a significance level of .05. Statistical analyses were performed using the software Statistical Package for the Social Sciences (SPSS version 25; SPSS Inc, Chicago, IL). Longitudinal data analyses and analyses using the zero-inflated negative binomial model were performed using SAS (version 9.4; SAS Institute, Cary, NC). Bootstrapping was performed using the Stata software (release 15; StataCorp LLC, College Station, TX). In agreement with the DSMB, no interim analysis was performed. The statistical analyses are described in more detail in the Statistical Analysis Plan (Appendix).

\section{Results}

\section{Participants and procedures}

Between April 27, 2012, and January 12, 2016, 645 women were registered as eligible, of whom 270 provided informed consent. Of the women who declined to be randomized, 295 women agreed to be followed up in an observational cohort study. Baseline characteristics of women randomized and women in the observational cohort were largely comparable (Supplemental Table 1).

The 270 women were randomly assigned to initial treatment with the LNG-IUS $(\mathrm{N}=132)$ or treatment with EA $(\mathrm{N}=138)$ (Figure 1). Baseline characteristics of the 2 treatment groups were comparable (Table 1).

Of the 132 women allocated to the LNG-IUS group, 122 (92\%) had an
LNG-IUS inserted, within a median time of 7 days from randomization (interquartile range $[\mathrm{IQR}], 0-21$ ) (Figure 1).

Of the 138 women allocated to the EA group, 130 (94\%) underwent the assigned intervention, within a median time of 27 days from randomization (IQR, 13-48) (Figure 1). In 7 of 130 women $(5.4 \%)$ who underwent EA, the ablation could not be completed (Supplemental Table 2).

\section{Primary outcome}

The PBAC score was obtained for 247 of 270 women (91\%) at 24 months' followup (Figure 1). The mean PBAC score was 64.8 in the LNG-IUS group vs 14.2 in the EA group (mean difference, 50.5; 95\% CI, 4.3-96.7) (Table 2; Supplemental Figure 1). As the upper limit of the 95\% CI exceeds the noninferiority margin of 25 points, noninferiority of the LNG-IUS could not be demonstrated (noninferiority, $P=87$ ). The $P$ value for the per-protocol analysis was identical (Supplemental Table 3).

Analysis of the primary outcome using a zero-inflated negative binomial model showed a significant difference in the PBAC score at 24 months for the negative binomial component, owing to 
FIGURE 2

PBAC scores at baseline and 3, 6, 12, and 24 months

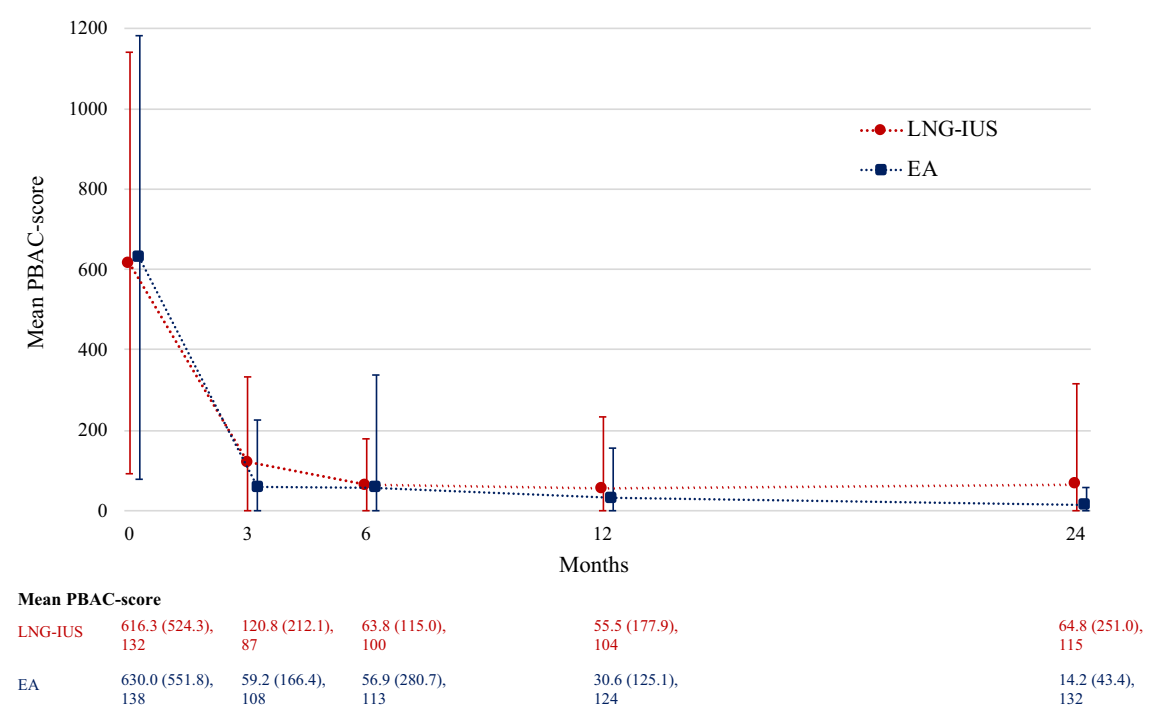

Intention-to-treat analysis. Mean PBAC scores (SD), total number of women shown at each time point. I bars indicate 95\% confidence intervals.

$E A$, endometrial ablation; LNG-IUS, levonorgestrel-releasing intrauterine system; $S D$, standard deviation.

Beelen et al. Levonorgestrel-releasing intrauterine system versus endometrial ablation for heavy menstrual bleeding. Am J Obstet Gynecol 2021.

some high scores in the LNG-IUS group (4.09; 95\% CI, 1.81-9.25) and a nonsignificant odds ratio for the proportion of women with zero scores $(0.78$; 95\% CI, 0.35-1.72).

A robust decline in PBAC scores in both groups was observed between baseline and 3 months' follow-up (Figure 2). At 3 months after randomization, $60 \%$ of women in the LNG-IUS group had reached a PBAC score not exceeding 75 points compared with $83 \%$ of women in the EA group (RR, 0.72; 95\% CI, 0.59-0.87) (Supplemental Figure 2). There was substantial variability in PBAC scores in the LNG-IUS group at 24 months; 4 women $(3.5 \%)$ still had a PBAC score of $>750$ points (Supplemental Figure 2). In 100 of 115 women (87\%) in the LNG-IUS group and 124 of 132 women (94\%) in the EA group, the PBAC score had decreased to $<75$ points (RR, 0.93; 95\% CI, 0.85-1.01) (Table 2; Supplemental Figure 2).

\section{Secondary outcomes}

No significant differences were found for secondary outcomes on menstruation pattern (amenorrhea, duration of blood loss, spotting, presence of clots, and dysmenorrhea) (Table 2).

The LNG-IUS was removed in 49 women $(39 \%)$ in the LNG-IUS group. Women who started with the LNG-IUS were more likely to receive a surgical or drug reintervention within 24 months compared with women in the EA group (44/127 [35\%] vs 27/138 [20\%]; RR, 1.77; 95\% CI, 1.17-2.68) (Table 3; Supplemental Figures 3 and 4). In the LNG-IUS group, 34 women (27\%) ultimately received a surgical intervention, 31 women (24\%) underwent EA, and 9 women $(7.1 \%)$ underwent a hysterectomy (of which 6 women had previously undergone EA). In the EA group, 14 women $(10 \%)$ underwent a hysterectomy. Most surgical reinterventions were performed within the first year after treatment $(26 / 34$ [76\%] in the LNG-IUS group and 10/14 [71\%] in the EA group) (Supplemental Figure 5). Additional drug treatment was prescribed to 19 women $(15 \%)$ in the LNG-IUS group vs $13(9.4 \%)$ in the EA group (RR, 1.59; 95\% CI, 0.82-3.08). The most common reason for a reintervention in both groups was persistence of HMB (Table 3).
Up to 12 months of follow-up, satisfaction rates were significantly higher in women in the EA group compared with women in the LNG-IUS group (Supplemental Table 4; Figure 3). At 24 months of follow-up, 74 of 100 women $(74 \%)$ in the LNG-IUS group were satisfied with their treatment compared with 98 of 116 women (84\%) in the EA group (RR, 0.88; 95\% CI, 0.76-1.01).

SF-36 scores were significantly lower over time in the LNG-IUS group for 4 of 9 domains (physical functioning, physical role, social functioning, and health change), compared with the EA group (between 2.3 and 7.6 points difference; scale of 0-100) (Supplemental Table 5). No significant differences were found in MMAS summary scores (mean difference, $3.3 ; 95 \% \quad \mathrm{CI}, \quad-0.5$ to 7.1 ) (Supplemental Table 6; Supplemental Figure 6) and in sexual function (FSFI) and distress (FSDS) scores over time (Supplemental Tables 7 and 8).

There were 13 SAEs in the LNG-IUS group and 8 SAEs in the EA group (Supplemental Table 9). Moreover, 1 SAE in the LNG-IUS group was possibly related to the intervention (tuboovarian abscess) vs 4 SAEs in the EA group (hematometra, endometritis, anaphylactic reaction on premedication, and observation for acute abdominal pain).

\section{Discussion}

\section{Principal findings of the study}

Both the strategy starting with an LNGIUS and the strategy starting with EA lead to a large decrease in menstrual blood loss, with comparable QoL and satisfaction scores. In most women in both the LNG-IUS and EA groups, the PBAC score decreased to a level not exceeding 75 points, indicating controlled bleeding. Nevertheless, there was a significant difference in the mean score, in favor of EA, and we could not demonstrate noninferiority of the LNGIUS strategy.

The LNG-IUS was removed in 39\% of the women in the LNG-IUS group within the 24 months of follow-up. The risk of receiving an additional intervention was almost twice as high in women who started with the LNG-IUS compared with women who started 
TABLE 3

Type and number of reinterventions after 24 months of follow-up

\begin{tabular}{|c|c|c|c|c|}
\hline Variable & LNG-IUS ( $n=127)$ & $E A(n=138)$ & Effect (RR) & $95 \% \mathrm{Cl}$ \\
\hline No. of women who discontinued the primary intervention $\mathrm{n}^{\mathrm{a}, \mathrm{b}}$ & $55(43.0)$ & $27(20.0)$ & 2.21 & $1.49-3.28$ \\
\hline No. of women with reinterventions ${ }^{b}$ & $44(35.0)$ & $27(20.0)$ & 1.77 & $1.17-2.68$ \\
\hline No. of women with LNG-IUS removal & $49(39.0)$ & $\mathrm{N} / \mathrm{A}$ & - & - \\
\hline No. of women with surgical reinterventions ${ }^{b}$ & $34(27.0)$ & $14(10.0)$ & 2.64 & $1.49-4.68$ \\
\hline Endometrial ablation & $31(24.0)$ & $1(0.7)$ & - & - \\
\hline First generation & $1(0.8)$ & $1(0.7)$ & - & - \\
\hline Second generation & $30(24.0)$ & $0(0.0)$ & - & - \\
\hline Hysterectomy & $9(7.1)$ & $14(10.0)$ & 0.70 & $0.31-1.56$ \\
\hline No. of women with drug reinterventions ${ }^{b}$ & $19(15.0)$ & $13(9.4)$ & 1.59 & $0.82-3.08$ \\
\hline \multicolumn{5}{|l|}{ Reason for reintervention ${ }^{\mathrm{c}, \mathrm{d}}$} \\
\hline HMB persisting & $42(63.0)$ & $18(53.0)$ & - & - \\
\hline Dysmenorrhea & $10(15.0)$ & $3(8.8)$ & - & - \\
\hline Pelvic pain & $8(12.0)$ & $3(8.8)$ & - & - \\
\hline Spotting & $7(10.0)$ & $1(2.9)$ & - & - \\
\hline Side effect & $15(22.0)$ & $1(2.9)$ & - & - \\
\hline Lost LNG-IUS & $8(12.0)$ & $\mathrm{N} / \mathrm{A}$ & - & - \\
\hline Hematometra & $1(1.5)$ & $1(2.9)$ & - & - \\
\hline Other ${ }^{\mathrm{e}}$ & $2(3.0)$ & $2(5.9)$ & - & - \\
\hline \multicolumn{5}{|l|}{ Values are given as number of women (percentage), unless otherwise indicated. } \\
\hline \multicolumn{5}{|c|}{$C l$, confidence interval; $E A$, endometrial ablation; $H M B$, heavy menstrual bleeding; $L N G-I U S$, levonorgestrel-releasing intrauterine system; $N / A$, not applicable; $R R$, relative risk. } \\
\hline \multicolumn{5}{|c|}{ 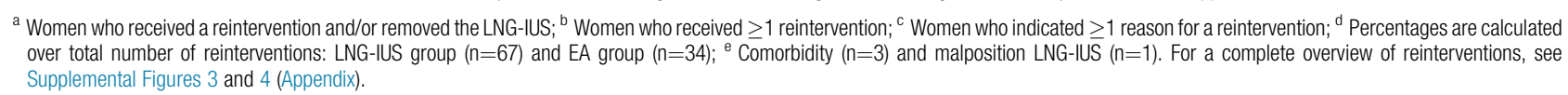 } \\
\hline
\end{tabular}

with EA. However, a surgical intervention was not performed in almost threequarters of the women in the LNG-IUS group, and hysterectomy rates did not differ significantly between the 2 treatment groups.

We found no differences over time between the 2 strategies in diseasespecific QoL and sexual functioning. General QoL scores were significantly better for 4 of 9 domains in the EA group; however, this difference was small (2.3-7.6 points on a 100 -point scale), making it debatable whether this difference is clinically relevant.

\section{Results}

In the first 6 months after treatment, a significantly larger percentage of women in the EA group were satisfied and had reached a level of controlled bleeding. This difference can be explained by the different mechanisms of action. Although EA works immediately after treatment, in the first months after insertion of the LNG-IUS, irregular bleeding and spotting are frequent side effects. ${ }^{21}$

The percentage of women in the LNGIUS group who underwent a surgical reintervention $(27 \%)$ was higher in our trial than in a trial in which the LNG-IUS was compared with oral medication (clinical effectiveness and costeffectiveness of levonorgestrel-releasing intrauterine system in primary care against standard treatment for menorrhagia [ECLIPSE] trial) (4\% EA and 6\% hysterectomy at 2 years), although the reported discontinuation rate was similar (36\% in the ECLIPSE trial and 39\% in our trial). ${ }^{3}$ The 5 -year follow-up of the ECLIPSE trial showed a doubling of surgical reintervention rates $(20 \%$ of women). ${ }^{33}$ The observed difference in surgical reintervention rates can be explained by the difference in comparative treatment. As in our trial, the LNGIUS was compared with EA; all participating women agreed to have 1 of these treatments, making them less inclined to preserve conservative treatment. Furthermore, the ECLIPSE trial was performed in a primary care setting, where EA is not the standard of care. Although in earlier research it was found that women prefer a treatment with no need for a repeat procedure, ${ }^{34}$ we did find comparable satisfaction rates at 24 months of follow-up.

\section{Research implications}

To investigate differences in costs between the 2 treatment strategies, we have planned to perform a cost-effectiveness analysis. Future research could further focus on prognostic factors that can 
FIGURE 3

Satisfaction rates at $3,6,12$, and 24 months

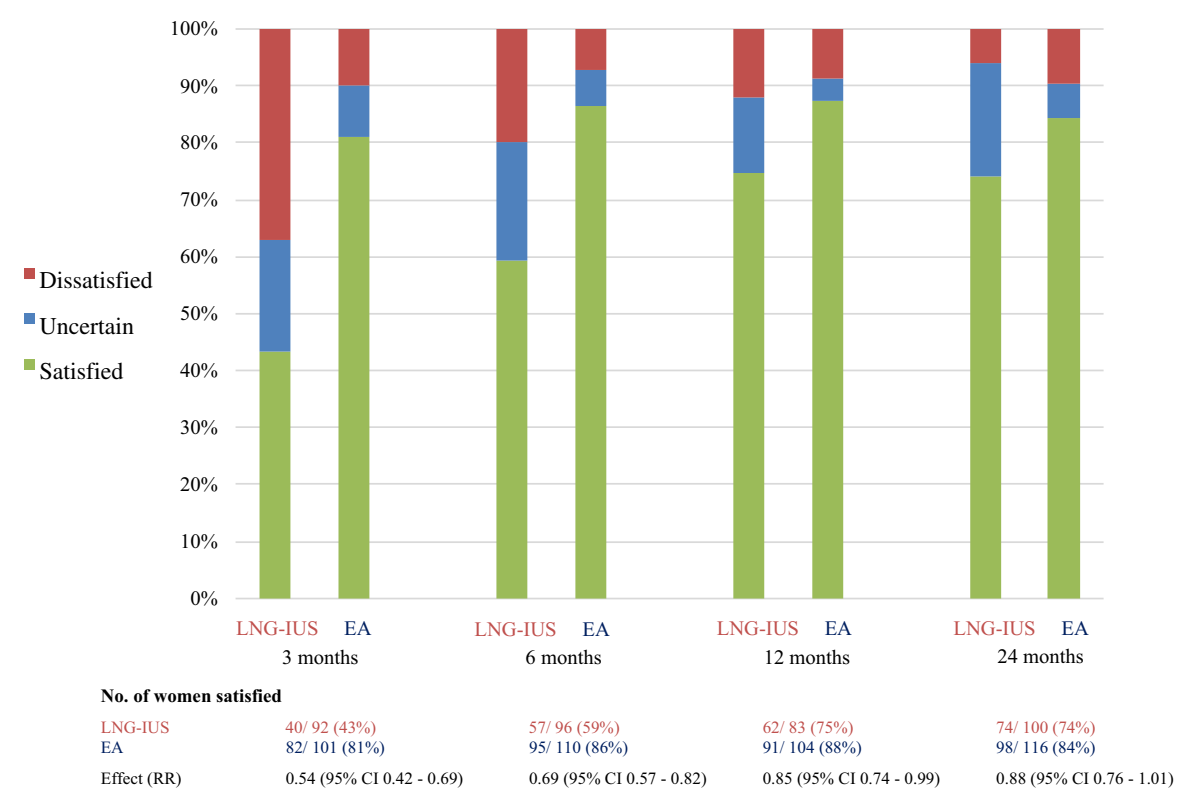

Intention-to-treat analysis. Number of women/total number of women (\%) and RR (95\% Cl) shown at each time point.

$\mathrm{Cl}$, confidence interval; $E A$, endometrial ablation; $L N G$-IUS, levonorgestrel-releasing intrauterine system; $R R$, relative risk.

Beelen et al. Levonorgestrel-releasing intrauterine system versus endometrial ablation for heavy menstrual bleeding. Am J Obstet Gynecol 2021.

predict failure of treatment with the LNG-IUS or EA and the construction of an individualized prediction model, allowing more personalized counseling of women with HMB while decreasing the chances of treatment failure.

\section{Strengths and limitations}

Key strengths of our trial are its multicenter design, with women recruited from both primary care and secondary care; a sample size larger than that of previous trials; the long follow-up period; and the relatively low number of women who were lost to follow-up for the primary outcome. The strategies evaluated in this pragmatic RCT reflect current practice, in which women can change or discontinue the initial treatment. Another strength is the limited set of exclusion criteria, making the results of this study generalizable to many women with HMB. Although a substantial number of women declined to be randomized, baseline characteristics of these women were largely comparable to the randomized women.
Because women were allowed to receive a reintervention during the follow-up period, all outcomes reflect the effect of a strategy, not just of the allocated intervention. Blinding of the participants was not possible, so decisions about reinterventions could be influenced by the initial preference and expectations of the participants and physicians.

Currently, there is no consensus about the preferable outcome measure in HMB studies, and a wide variety of treatment outcomes are being used. ${ }^{35}$ The most frequently used measures are blood loss-related outcome measures. ${ }^{35,36}$ Our primary outcome measure was the PBAC score, which was found to be significantly associated with satisfaction and the chance of receiving a reintervention. ${ }^{37}$ As HMB has a major impact on women's well-being, we thought it important to pay special attention to QoL and sexual functioning as well. By combining the PBAC score with these secondary outcomes, we aimed to generate a broad assessment of the effectiveness of both treatments. ${ }^{38}$ At the moment, the Core Outcome Measures in Effectiveness Trials initiative is working on a core outcome set for HMB that covers all aspects of the condition, which can hopefully be used in future research. ${ }^{39}$

\section{Conclusions and clinical implications}

This study showed that a strategy starting with the LNG-IUS and a strategy starting with EA lead to controlled bleeding in most women with comparable satisfaction, QoL, and sexual function scores. With both strategies, a large decrease in menstrual blood loss was found. Nevertheless, the mean score at 24 months was lower in the EA group, and we could not demonstrate noninferiority of the LNG-IUS strategy. Women who started with the LNG-IUS more frequently had further treatment, with a quarter of the women undergoing EA.

The results of this study will enable physicians to provide women with clear information about multiple important outcomes after HMB treatment. For instance, the faster reduction in menstrual blood loss and lower chance of needing additional treatment of EA can be weighed against the less-invasive and reversible characteristics of the LNGIUS. On the basis of the treatment outcomes reported here, women with $\mathrm{HMB}$ should be able to make a well-informed decision about treatment.

\section{Acknowledgments}

We thank all the women who participated in this trial; the participating general practitioners, gynecologists, and hospitals; the research nurses; and the staff of the Dutch Consortium for Studies in Women's Health and Reproduction.

\section{References}

1. Fraser IS, Mansour D, Breymann C, Hoffman C, Mezzacasa A, Petraglia F. Prevalence of heavy menstrual bleeding and experiences of affected women in a European patient survey. Int J Gynaecol Obstet 2015;128: 196-200.

2. National Institute for Health and Care Excellence. Heavy menstrual bleeding: assessment and management. NICE guideline [NG88]. 2018. Available at: https://www. nice.org.uk/guidance/ ng88. Accessed August 21, 2019. 
3. Gupta J, Kai J, Middleton L, et al. Levonorgestrel intrauterine system versus medical therapy for menorrhagia. N Engl J Med 2013;368 128-37.

4. Lethaby A, Hussain M, Rishworth JR, Rees MC. Progesterone or progestogenreleasing intrauterine systems for heavy menstrual bleeding. Cochrane Database Syst Rev 2015;4:CD002126.

5. Daud S, Ewies AA. Levonorgestrel-releasing intrauterine system: why do some women dislike it? Gynecol Endocrinol 2008;24:686-90.

6. Marjoribanks J, Lethaby A, Farquhar C. Surgery versus medical therapy for heavy menstrual bleeding. Cochrane Database Syst Rev 2016;2016:CD003855.

7. Smith PP, Malick S, Clark TJ. Bipolar radiofrequency compared with thermal balloon ablation in the office: a randomized controlled trial. Obstet Gynecol 2014;124:219-25.

8. Penninx JP, Herman MC, Mol BW, Bongers MY. Five-year follow-up after comparing bipolar endometrial ablation with hydrothermablation for menorrhagia. Obstet Gynecol 2011;118:1287-92.

9. Bongers MY. Hysteroscopy and heavy menstrual bleeding (to cover TCRE and secondgeneration endometrial ablation). Best Pract Res Clin Obstet Gynaecol 2015;29:930-9.

10. Bofill Rodriguez M, Lethaby A, Grigore M, Brown J, Hickey M, Farquhar C. Endometrial resection and ablation techniques for heavy menstrual bleeding. Cochrane Database Syst Rev 2019;1:CD001501.

11. Soysal M, Soysal S, Ozer S. A randomized controlled trial of levonorgestrel releasing IUD and thermal balloon ablation in the treatment of menorrhagia. Zentralbl Gynakol 2002;124: 213-9.

12. Tam WH, Yuen PM, Shan Ng DP, Leung PL, Lok $I H$, Rogers MS. Health status function after treatment with thermal balloon endometrial ablation and levonorgestrel intrauterine system for idiopathic menorrhagia: a randomized study. Gynecol Obstet Invest 2006;62:84-8.

13. de Souza SS, Camargos AF, de Rezende CP, Pereira FA, Araújo CA, Silva Filho AL. A randomized prospective trial comparing the levonorgestrel-releasing intrauterine system with thermal balloon ablation for the treatment of heavy menstrual bleeding. Contraception 2010;81:226-31.

14. Shaw RW, Symonds IM, Tamizian O, Chaplain J, Mukhopadhyay S. Randomised comparative trial of thermal balloon ablation and levonorgestrel intrauterine system in patients with idiopathic menorrhagia. Aust N Z J Obstet Gynaecol 2007:47:335-40.

15. Busfield RA, Farquhar CM, Sowter MC, et al. A randomised trial comparing the levonorgestrel intrauterine system and thermal balloon ablation for heavy menstrual bleeding. BJOG 2006;113: 257-63.

16. Barrington JW, Arunkalaivanan AS, AbdelFattah M. Comparison between the levonorgestrel intrauterine system (LNG-IUS) and thermal balloon ablation in the treatment of menorrhagia. Eur J Obstet Gynecol Reprod Biol 2003;108:72-4.

17. Silva-Filho AL, Pereira Fde A, de Souza SS, et al. Five-year follow-up of levonorgestrelreleasing intrauterine system versus thermal balloon ablation for the treatment of heavy menstrual bleeding: a randomized controlled trial. Contraception 2013;87:409-15.

18. Ghazizadeh S, Panahi Z, Ghanbari Z, Menshadi AT, Farahmandian T, Javadian P. Comparative efficacy of NovaSure, the levonorgestrel-releasing intrauterine system, and hysteroscopic endometrial resection in the treatment of menorrhagia: a randomized clinical trial. J Gynecol Surg 2014;30:215-8.

19. Middleton $L J, \quad C h a m p a n e r i a \quad R$, Daniels JP, et al. Hysterectomy, endometrial destruction, and levonorgestrel releasing intrauterine system (Mirena) for heavy menstrual bleeding: systematic review and metaanalysis of data from individual patients. BMJ 2010;341:c3929.

20. Herman MC, van den Brink MJ, Geomini PM, et al. Levonorgestrel releasing intrauterine system (Mirena) versus endometrial ablation (Novasure) in women with heavy menstrual bleeding: a multicentre randomised controlled trial. BMC Womens Health 2013;13: 32.

21. Bayer HealthCare Pharmaceuticals. Product information: Mirena. 2017. Available at: https:// resources.bayer.com.au/resources/uploads/pi/ file9399.pdf. Accessed December 23, 2019.

22. Food and Drug Administration. Summary of safety and effectiveness data: NovaSure ${ }^{\mathrm{TM}}$ impedance controlled endometrial ablation system. 2019. Available at: https://www. accessdata.fda.gov/cdrh_docs/pdf/P010013b. pdf. Accessed December 23, 2019.

23. Bujold K. NovaSure ${ }^{\circledR}$ instructions for use and controller operator's manual. 2018. Available at: https://www.hologic.com/sites/default/ files/2018-02/MAN-03523-001_009_01_0_0. pdf. Accessed June 14, 2020.

24. Higham JM, O'Brien PM, Shaw RW. Assessment of menstrual blood loss using a pictorial chart. Br J Obstet Gynaecol 1990;97: 734-9.

25. Shaw RW, Brickley MR, Evans L, Edwards MJ. Perceptions of women on the impact of menorrhagia on their health using multi-attribute utility assessment. $\mathrm{Br} J$ Obstet Gynaecol 1998;105:1155-9.

26. Pattison H, Daniels JP, Kai J, Gupta JK. The measurement properties of the menorrhagia multi-attribute quality-of-life scale: a psychometric analysis. BJOG 2011;118:1528-31.

27. Garratt AM, Ruta DA, Abdalla MI, Buckingham JK, Russell IT. The SF36 health survey questionnaire: an outcome measure suitable for routine use within the NHS? BMJ 1993;306:1440-4.

28. Clark TJ, Khan KS, Foon R, Pattison $H$, Bryan S, Gupta JK. Quality of life instruments in studies of menorrhagia: a systematic review. Eur J Obstet Gynecol Reprod Biol 2002;104: 96-104.
29. Derogatis $L R$, Rosen $R$, Leiblum $S$, Burnett A, Heiman J. The Female Sexual Distress Scale (FSDS): initial validation of a standardized scale for assessment of sexually related personal distress in women. J Sex Marital Ther 2002;28:317-30.

30. Rosen R, Brown C, Heiman J, et al. The Female Sexual Function Index (FSFI): a multidimensional self-report instrument for the assessment of female sexual function. J Sex Marital Ther 2000;26:191-208.

31. Crosignani $P G$, Vercellini $P$, Mosconi $P$, Oldani S, Cortesi I, De Giorgi O. Levonorgestrelreleasing intrauterine device versus hysteroscopic endometrial resection in the treatment of dysfunctional uterine bleeding. Obstet Gynecol 1997:90:257-63.

32. Istre $O$, Trolle B. Treatment of menorrhagia with the levonorgestrel intrauterine system versus endometrial resection. Fertil Steril 2001;76:304-9.

33. Kai J, Middleton L, Daniels J, et al. Usual medical treatments or levonorgestrel-IUS for women with heavy menstrual bleeding: longterm randomised pragmatic trial in primary care. Br J Gen Pract 2016;66:e861-70.

34. van den Brink MJ, Beelen $P$, Herman MC, et al. Women's preferences for the levonorgestrel intrauterine system versus endometrial ablation for heavy menstrual bleeding. Eur J Obstet Gynecol Reprod Biol 2018:228:143-7.

35. Herman MC, Penninx J, Geomini PM, Mol BW, Bongers MY. Choice of primary outcomes evaluating treatment for heavy menstrual bleeding. BJOG 2016;123:1593-8.

36. Bergeron $C$, Laberge $P Y$, Boutin $A$, et al. Endometrial ablation or resection versus levonorgestrel intra-uterine system for the treatment of women with heavy menstrual bleeding and a normal uterine cavity: a systematic review with meta-analysis. Hum Reprod Update 2020;26: 302-11.

37. Herman MC, Mak N, Geomini PM, et al. Is the Pictorial Blood Loss Assessment Chart (PBAC) score associated with treatment outcome after endometrial ablation for heavy menstrual bleeding? A cohort study. BJOG 2017; 124:277-82.

38. Magnay JL, O'Brien S, Gerlinger C, Seitz C. A systematic review of methods to measure menstrual blood loss. BMC Womens Health 2018;18:142.

39. Cooper NAM, Khan K. Defining core outcomes for clinical trials of heavy menstrual bleeding: a Core Outcome Sets for Gynaecological Conditions (COGS) project. Comet Initiative. 2019. Available at: http://www.cometinitiative.org/studies/details/789. Accessed December 19, 2019.

\section{Author and article information}

From the Department of Obstetrics and Gynaecology, Máxima MC, Veldhoven, Netherlands (Drs Beelen, Geomini, Coppus, and Bongers); Department of General Practice and Elderly Care Medicine, University of 
Groningen, University Medical Centre Groningen, Groningen, Netherlands (Drs van den Brink, Dekker, and Berger); Department of Obstetrics and Gynaecology, Academic Medical Centre, Amsterdam, Netherlands (Drs Duijnhoven, van Meurs, and Timmermans); Department of General Practice, University of Maastricht, Maastricht, Netherlands (Drs Beelen and Mak); Department of Obstetrics and Gynaecology, Jeroen Bosch Hospital, 'sHertogenbosch, Netherlands (Drs Herman and van der Steeg); Department of Obstetrics and Gynecology, Gelre Hospital, Apeldoorn, Netherlands (Dr Eising); Department of Obstetrics and Gynaecology, Medisch Spectrum Twente, Twente, Netherlands (Dr Massop-Helmink); Department of Obstetrics and Gynaecology, University Medical Centre Groningen, Groningen, Netherlands (Dr Klinkert); Department of Obstetrics and Gynaecology, Radboud University Medical Centre, Nijmegen, Netherlands (Dr Nieboer); Department of Obstetrics and Gynecology, Deventer Hospital, Deventer, Netherlands (Dr van der Voet); Department of Reproductive Medicine and Gynecology, University Medical Center Utrecht, Utrecht, Netherlands (Dr Veersema); Department of
Obstetrics and Gynecology, Zuyderland Medical Centre, Heerlen, Netherlands (Dr Smeets); Department of Obstetrics and Gynecology, Isala Hospital, Zwolle, Netherlands (Dr Schutte); Department of Obstetrics and Gynecology, Flevo Hospital, Almere, Netherlands (Dr Baal); Department of Clinical Epidemiology, Biostatistics and Bioinformatics, Academic Medical Centre, Amsterdam, Netherlands (Dr Bossuyt); Department of Obstetrics and Gynaecology, Monash University, Melbourne, Victoria, Australia (Dr Mol); and Research School Grow, University of Maastricht, Maastricht, Netherlands (Dr Bongers).

${ }^{1}$ These authors contributed equally to this work.

Received March 12, 2020; revised June 30, 2020; accepted Aug. 10, 2020

B.W.J.M. reports receiving grants from Guerbet and Merck; personal fees from Guerbet, Merck, and ObsEva; and other financial relationships with Guerbet (travel support to present at meetings), outside the submitted work. M.Y. Bongers reports other financial relationships with Gynesonics (device reduction in clinical trials), outside the submitted work. M.Y. Bongers is a member of the communication team of Bayer and the advisory board of Hologic. The remaining authors report no conflict of interest.

This trial was funded by the Netherlands Organization for Health Research and Development (ZonMw, grant number 171202001). The funder of the study had no role in the study design, collection, analysis, and interpretation of the data; in the writing of the manuscript; and in the decision to submit the article for publication. The authors had full access to all the data in the study and had final responsibility for the decision to submit for publication.

The study was registered in the Netherlands Trial Register before enrollment with the clinical trial identification number NL2842 (https://www. trialregister.nl). The date of registration was July 12,2011 . The date of initial participant enrollment was April 27, 2012.

The results of the study were presented at the Royal College of Obstetricians and Gynaecologists World Congress, London, United Kingdom, June 19, 2019.

Corresponding author: Pleun Beelen, MD. pleun. beelen@gmail.com 